\title{
Aplicación de un Ciclo de Mejora Docente en "Dinámica de la Partícula"
}

\author{
ALEJANDRO JAVIER MARTÍNEZ ROS \\ Universidad de Sevilla \\ Dpto. de Física Aplicada I \\ amartinez49@us.es \\ ORCID: https://orcid.org/0000-0001-7277-3326 \\ D.O.I.: http://dx.doi.org/10.12795/JDU.2018.i01.64 \\ Pp.: $1135-1152$
}

\section{Resumen}

En esta comunicación se describe el diseño, aplicación y evaluación de un ciclo de mejora docente (CMD) realizado en la asignatura de Física I del Grado de Ingeniería Electrónica Industrial de la Universidad de Sevilla. En concreto, el ciclo se ha llevado a cabo durante el Tema 3 de la asignatura que trata sobre dinámica de la partícula. A lo largo de todo el ciclo se ha buscado conseguir un aprendizaje crítico por parte de los alumnos. Para ello, se han diseñado actividades con el objetivo de que los alumnos se replantearan conceptos previamente establecidos y fueran capaces de evaluar sus conocimientos. Por otro lado, también se ha intentado fomentar el trabajo en grupo mediante la realización de un taller basado en la resolución de un problema. Por último, el progreso de aprendizaje a lo largo del ciclo se ha evaluado mediante el uso de un cuestionario inicial y final. 
Palabras clave: Física I, Ingeniería Electrónica Industrial, docencia universitaria y experimentación docente universitaria.

\section{Contexto}

La experiencia docente se ha llevado a cabo en la asignatura de Física I del Grado Ingeniería Electrónica Industrial de la Universidad de Sevilla. Esta asignatura es de primer curso y suele ser la primera toma de contacto de los alumnos con la universidad. La asignatura cuenta con 5 créditos de teoría y 1 de prácticas. Esto implica que las clases de teoría se distribuyen a lo largo de la semana de la siguiente manera: 4 horas de clase durante las 5 primeras semanas y el resto del cuatrimestre 3 horas a la semana.

La asignatura está dividida en tres grupos de teoría y los exámenes de convocatoria suelen ser comunes, en particular, el grupo de teoría 2 que es donde se ha desarrollado el ciclo de mejora consta de 68 alumnos matriculados, de los cuales 24 son repetidores y no suelen asistir a clase. Por otro lado, aunque la gran mayoría de los alumnos sí que han cursado la asignatura de Física durante su formación en la educación secundaria, hay algunos que provienen de formación profesional o de la rama sanitaria y tienen conocimientos previos de la asignatura más básicos.

En cuanto al ciclo de mejora, se realizó durante la primera mitad del cuatrimestre del curso 2018/2019 y estuvo centrado en el tema 3 de "Dinámica de la Partícula". Debido a la distribución del aula, que cuenta con bancos fijos para los estudiantes y una tarima donde se sitúa la mesa del profesor, se ve limitado el libre movimiento de los alumnos y del profesor para desarrollar algunas actividades. 


\section{Diseño previo del Ciclo de Mejora Docente}

El modelo metodológico que había seguido hasta la fecha consistía en una exposición teórica y una parte de realización de problemas al final del tema, ver Figura 1.

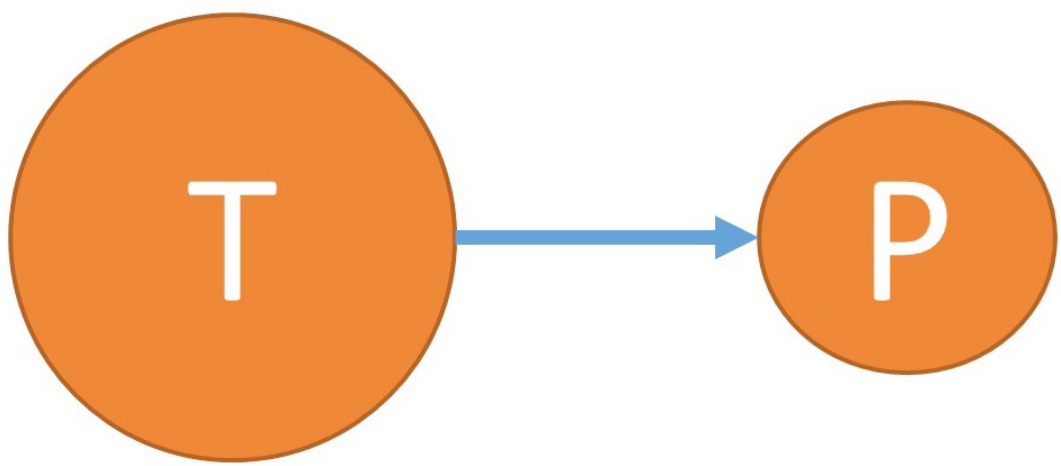

Figura 1: Modelo metodológico previo.

El problema fundamental que se observa en este tipo de modelo es que los alumnos no participan en el aprendizaje de forma continuada, ya que al final solo se dedican a estudiar para los parciales y para el examen final. Por ello, he intentado modificar mi modelo metodológico asumiendo los siguientes principios didácticos:

a) Aprendizaje del alumnado: enfoque centrado en el alumno y desarrollo de un ambiente de aprendizaje crítico, buscando una mejor motivación de los estudiantes y un mayor interés por la asignatura. También se ha buscado obtener una retroalimentación continuada de su aprendizaje mediante problemas al final del tema y cuestionarios. De esta forma se pueden corregir posibles desviaciones respecto a lo esperado.

b) Contenidos de enseñanza: organización de los contenidos mediante el uso de mapas. Con este tipo de herramientas se pueden apreciar de una manera muy visual y directa las diferentes relaciones que existen entre los contenidos que 
pretendemos que los alumnos aprendan, así como ayudarnos en el diseño de la secuencia de actividades.

c) Metodología: se ha buscado organizar los contenidos y las secuencias de actividades alrededor de preguntas clave para lograr una mejor motivación del alumnado. Otro aspecto clave en el cambio metodológico propuesto consiste en lograr que los alumnos sean los que trabajen y aprendan por ellos mismos, en lugar de que sea el profesor el que les transmita toda la información. De esta forma se busca favorecer un aprendizaje más duradero de los contenidos.

d) Evaluación: el diagnóstico inicial y final de las ideas o modelos mentales de los estudiantes es otro elemento que resulta muy útil para mejorar la docencia. De esta forma se puede ver dónde tienen mayores dificultades en los contenidos, ya sea por falta de conocimientos y/o porque los conceptos no están bien asimilados, y trabajar para corregirlo. Además, se ha buscado llevar un seguimiento continuado acerca del aprendizaje de los alumnos mediante la información aportada en los diferentes problemas que se han realizado en el aula.

Partiendo de los principios didácticos mencionados y con el objetivo de lograr que los alumnos tengan un mayor interés por la asignatura, he planteado el siguiente modelo metodológico (mostrado en la Figura 2), en el que la idea fundamental consiste en lograr un aprendizaje más continuo y un mayor interés por la asignatura.

Jornadas de Formación e Innovación Docente del Profesorado | № 1 (2018) Esta obra se distribuye con la licencia Creative Commons 
Modelo Metodológico Posible

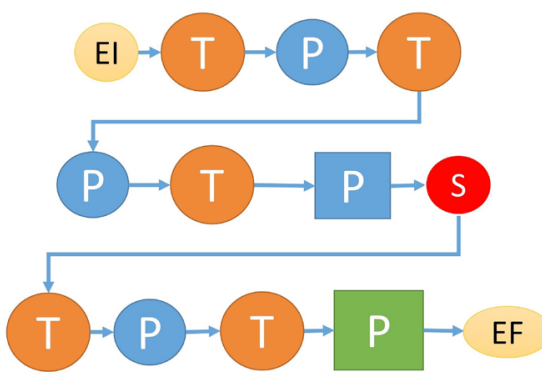

EF Exploración final

El Exploración inicial

S Sondeo de aprendizaje

Exposición teórica

P Resolución de problemas por parte de los alumnos

P Resolución de problemas por parte del profesor

P Resolución de problemas por parte de los alumnos con evaluación

Figura 2. Modelo metodológico posible.

Este modelo metodológico fue implementado durante el ciclo de mejora y se desarrolló de la siguiente manera: en primer lugar se les pasó a los alumnos un cuestionario inicial, El, para valorar sus conocimientos previos sobre el tema que íbamos a tratar, de esa forma pude hacer una mejor valoración sobre dónde tenían una mayor falta de conocimientos y readaptar las actividades en función de ello. Una vez analizado el cuestionario inicial, les expuse a los alumnos los conceptos teóricos, T, que necesitaban en cada parte del tema, partiendo de una serie de preguntas clave: ¿Por qué se mueven los cuerpos?, ¿Cómo se relacionan las fuerzas con los cuerpos?, ¿Cómo se generan las fuerzas? y ¿Cómo se utilizan para resolver problemas?, todo ello con el objetivo de que fuesen los alumnos los que dedujeran esos conceptos.

Después, se les mostró a los alumnos cómo resolver algunos problemas escogidos, $\mathrm{P}$, relacionados con los conceptos que estábamos viendo y que hacian hincapié en las dificultades encontradas en el cuestionario inicial. También se les propusieron algunos problemas para que los intentaran resolver en casa. La idea era que ellos se enfrentaran primero a los problemas para comprobar dónde estaban sus dificultades y resolverlas. Después de cada problema se realizó un sondeo, S, para comprobar si habían adquirido los conceptos tratados y así, función del resultado, ir modificando las explicaciones o, en caso necesario, resolverlo yo mismo. 
Otra actividad importante realizada siguiendo este modelo metodológico ha consistido en un taller basado en un problema que tenían que resolver por parejas y que se valoraba para la evaluación final. El objetivo de este taller era que los alumnos aprendiesen a razonar entre ellos y a contrastar lo que habían aprendido (Finkel, 2008). Por último, se volvió a pasar el cuestionario inicial para poder realizar una evaluación sobre los conocimientos que habían adquirido al final del ciclo (Porlán, 2017).

\section{Mapa de contenidos}

Para ayudarme en la organización de los contenidos tratados en el ciclo, he utilizado el mapa que se muestra en la Figura 3, en el cual se establecen las principales relaciones que existen entre los contenidos del tema seleccionado: Dinámica de la partícula. En concreto se puede ver cómo se han dispuesto tres contenidos conceptuales organizadores (Fuerza, Masa y Leyes de Newton) y cómo se relacionan con el resto de contenidos conceptuales (en color azul). Además, también he buscado relacionar los contenidos procedimentales (en color verde), útiles en la resolución de problemas, con el resto de contenidos conceptuales. Por último, también he pretendido acompañar todos los contenidos que se trabajan en el tema con unos contenidos actitudinales que se van a abordar durante todo el curso, como son: el aprendizaje crítico y la valoración del aprendizaje continuo.

Como se puede ver en la Figura 3, el desarrollo del ciclo de mejora ha girado en torno a cuatro preguntas clave, las cuales intentaban abarcar los principales contenidos tratados en el tema (Tipler-Mosca, 2017). Además, para su elección también he procurado que fueran motivantes para los estudiantes y que se pudieran ir respondiendo conforme se iba avanzando en el tema. En concreto, las preguntas escogidas fueron las siguientes:

- ¿Por qué se mueven los cuerpos?

- ¿Cómo se generan las fuerzas?

- ¿Cómo se relacionan las fuerzas con los cuerpos?

- ¿Cómo se utilizan para resolver problemas? 


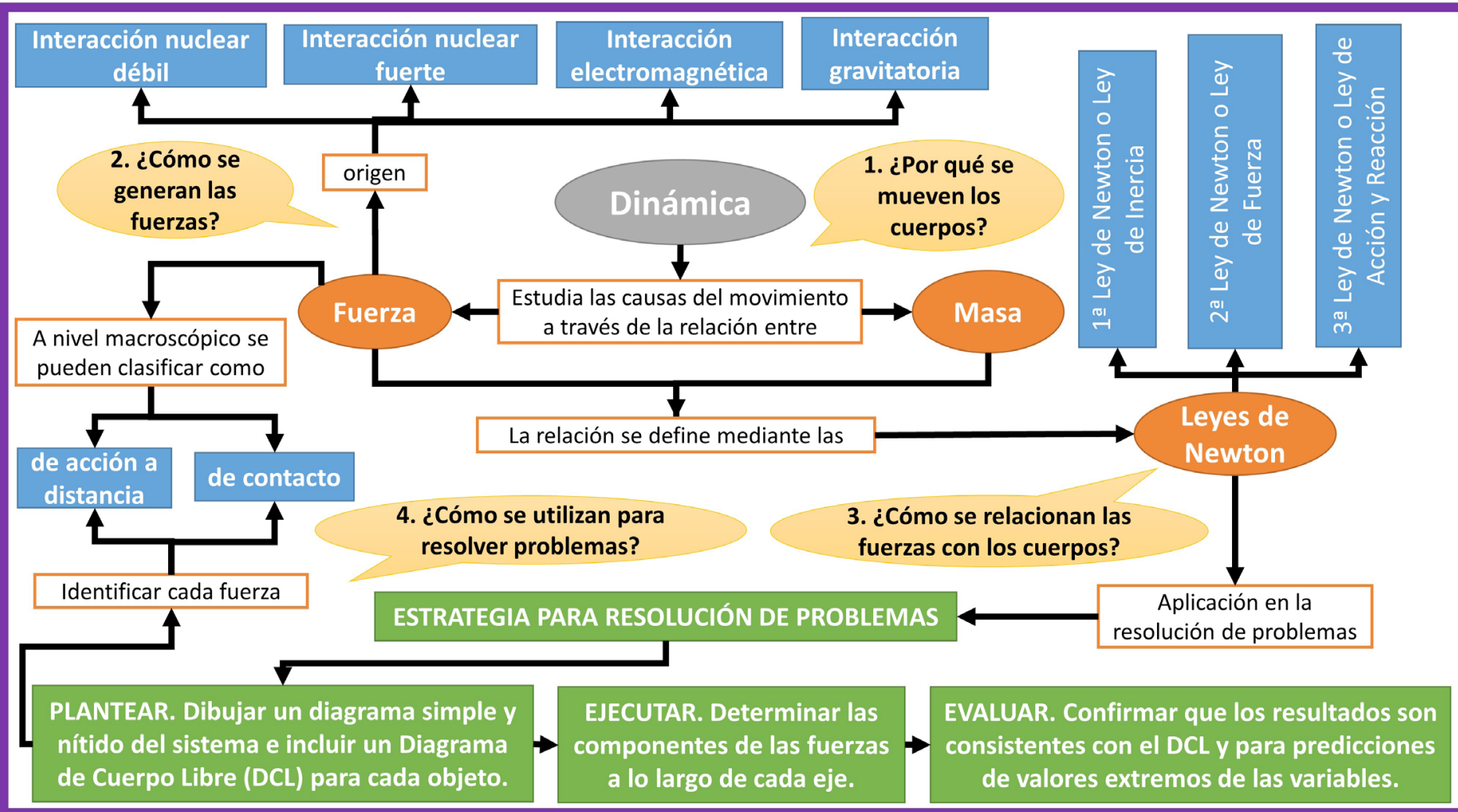

Figura 3. Mapa de contenidos.

Jornadas de Formación e Innovación Docente del Profesorado | № 1 (2018)

cc (7) $\Theta$ Esta obra se distribuye con la licencia Creative Commons 


\section{Exploración de ideas previas}

Para acceder a los conocimientos previos del alumno y poder evaluar su progreso de aprendizaje al inicio y al final del ciclo de mejora, se ha usado un cuestionario de ideas previas (Porlán, 2017).

Las preguntas del cuestionario inicial y final han sido las siguientes:

1. Suponga que estamos empujando un bloque muy pesado y no hay rozamiento con el suelo. Después de un tiempo dejamos de empujar ¿qué le sucederá al bloque, se detendrá o continuará moviéndose de manera indefinida?

2. Suponga que usted es un ingeniero que trabaja en la ESA y debe diseñar una sonda para aterrizar en Mercurio, para ello es fundamental conocer la masa de la sonda que llegará al planeta. Se sabe que un cuerpo pesa 3,5 veces más en la Tierra que en Mercurio ¿cuál sería entonces la relación entre la masa del cuerpo en ambos planetas?

3. Suponga que estamos en el antiguo Egipto y queremos mover un bloque de piedra, para ello le atamos una cuerda y tiramos. Si sabemos que la tercera ley de Newton, conocida como de acción-reacción, nos dice que si aplico una fuerza sobre un cuerpo este cuerpo ejerce la misma fuerza sobre mí, pero en sentido contrario. ¿Cómo es posible entonces que podamos mover el bloque?

4. Explique en cuál de las siguientes situaciones la fuerza neta sobre el cuerpo es cero:

a) un avión que vuela al norte con rapidez constante de $120 \mathrm{~m} / \mathrm{s}$ y altitud constante.

b) un automóvil que sube en línea recta por una colina con pendiente de $3^{\circ}$, a una rapidez constante de $90 \mathrm{~km} / \mathrm{h}$. 
c) un halcón que se mueve en círculos con rapidez constante de $20 \mathrm{~km} / \mathrm{h}$ a una altura constante de 15 m sobre un campo abierto.

d) una caja con superficies lisas, sin fricción, que está en la parte de atrás de un camión cuando éste acelera hacia adelante en un camino plano a $5 \mathrm{~m} / \mathrm{s}^{2}$.

5. Suponga que una astronauta llega a un planeta donde g=19.6 m/s2. En comparación con la Tierra, ¿le sería más fácil, más difícil o igual de fácil caminar ahí? ¿Le sería más fácil, más difícil o igual de fácil atrapar una pelota que se mueve horizontalmente hacía él a $12 \mathrm{~m} / \mathrm{s}$ ? (Suponga que el traje espacial es un modelo ligero que no impide en absoluto los movimientos de la astronauta.)

Como se puede apreciar, en este cuestionario se trataban, de una manera general, los principales conceptos tratados en el tema de Dinámica de la partícula, ver Figura 3. En particular, se ha buscado comprobar sus conocimientos sobre las leyes de Newton, las cuales se preguntan de una manera indirecta en el cuestionario. Por ejemplo, para responder a la pregunta 1 es necesario conocer la primera ley de Newton, mientras que para responder a la segunda es necesario saber el significado del concepto de masa. Por otro lado, en la pregunta 3 se ha buscado saber sus conocimientos sobre la tercera ley de Newton, mientras que en las preguntas 4 y 5 se necesitaba una combinación de varias leyes y del concepto de masa.

\section{Aplicación del CMD}

A continuación se muestra un relato resumido de las sesiones y se describe el desarrollo de cada una de las actividades del ciclo de mejora. En primer lugar, en la Tabla 1 , se describen las actividades llevadas a cabo a lo largo del ciclo y el objetivo marcado para cada una de ellas. 
Después, en la Tabla 2, se muestra de una manera resumida como se desarrollaron cada una de las actividades del ciclo de mejora.

Tabla 1. Secuencia de actividades y desarrollo del ciclo de mejora.

\begin{tabular}{|c|c|c|c|}
\hline$\#$ & Actividades & $\begin{array}{c}\text { Tiempo } \\
\text { (min) }\end{array}$ & Objetivo \\
\hline 1 & Cuestionario Inicial & 20 & $\begin{array}{l}\text { Conocer si tienen conocimientos } \\
\text { previos sobre el tema. }\end{array}$ \\
\hline 2 & $\begin{array}{l}\text { Exposición: ¿Por qué se } \\
\text { mueven los cuerpos? }\end{array}$ & 20 & $\begin{array}{l}\text { Que conozcan de manera razo- } \\
\text { nada qué es una fuerza. }\end{array}$ \\
\hline 3 & $\begin{array}{l}\text { Ejemplo de uso de super- } \\
\text { posición de fuerzas }\end{array}$ & 15 & $\begin{array}{l}\text { Que comprendan el carácter vec- } \\
\text { torial de la fuerza y que aprendan } \\
\text { a obtener la fuerza resultante en } \\
\text { un sistema. }\end{array}$ \\
\hline 4 & $\begin{array}{l}\text { Exposición: ¿Cómo se ge- } \\
\text { neran las fuerzas? }\end{array}$ & 40 & $\begin{array}{l}\text { Que conozcan cuales son las in- } \\
\text { teracciones que producen fuerzas. }\end{array}$ \\
\hline 5 & $\begin{array}{l}\text { Exposición: ¿Cómo se re- } \\
\text { lacionan las fuerzas con } \\
\text { los cuerpos? (1ạ ley de } \\
\text { Newton) }\end{array}$ & 30 & $\begin{array}{l}\text { Que conozcan la 1a ley de Newton } \\
\text { y el significado de sistema de re- } \\
\text { ferencia inercial. }\end{array}$ \\
\hline 6 & $\begin{array}{l}\text { Ejemplo de cómo usar la } \\
\text { 1a ley de Newton y plan- } \\
\text { tear un problema para } \\
\text { resolver en casa sobre } \\
\text { sistemas de referencia } \\
\text { inercial }\end{array}$ & 10 & $\begin{array}{l}\text { Que aprendan a aplicar la } 1 \underline{a} \\
\text { ley de Newton y enfrentar a } \\
\text { los alumnos a la resolución de } \\
\text { problemas. }\end{array}$ \\
\hline 7 & $\begin{array}{l}\text { Exposición: ¿Cómo se re- } \\
\text { lacionan las fuerzas con } \\
\text { los cuerpos? (2a ley de } \\
\text { Newton) }\end{array}$ & 25 & $\begin{array}{l}\text { Que conozcan la 2a ley de Newton } \\
\text { y el concepto de masa. }\end{array}$ \\
\hline 8 & $\begin{array}{l}\text { Exposición: ¿Cómo se re- } \\
\text { lacionan las fuerzas con } \\
\text { los cuerpos? (3a- ley de } \\
\text { Newton) }\end{array}$ & 15 & $\begin{array}{l}\text { Que conozcan la 3a ley de } \\
\text { Newton. }\end{array}$ \\
\hline 9 & $\begin{array}{l}\text { Exposición: ¿Cómo se uti- } \\
\text { lizan para resolver pro- } \\
\text { blemas? (Fuerzas a } \\
\text { distancia) }\end{array}$ & 25 & $\begin{array}{l}\text { Que conozcan otra clasificación } \\
\text { alternativa para las fuerzas se- } \\
\text { gún su rango de acción. Fuerzas a } \\
\text { distancia. }\end{array}$ \\
\hline
\end{tabular}




\begin{tabular}{|c|c|c|c|}
\hline 10 & $\begin{array}{l}\text { Ejemplo sobre fuerza de } \\
\text { acción a distancia }\end{array}$ & 10 & $\begin{array}{l}\text { Que comprendan el concepto de } \\
\text { fuerza gravitatoria. Peso. }\end{array}$ \\
\hline 11 & $\begin{array}{l}\text { Exposición: ¿Cómo se uti- } \\
\text { lizan para resolver pro- } \\
\text { blemas? (Fuerzas de } \\
\text { contacto) }\end{array}$ & 30 & $\begin{array}{l}\text { Que conozcan los tipos de fuerzas } \\
\text { de contacto que hay. }\end{array}$ \\
\hline 12 & $\begin{array}{l}\text { Ejemplo sobre fuerza de } \\
\text { contacto }\end{array}$ & 15 & $\begin{array}{l}\text { Que comprendan como intervie- } \\
\text { nen las fuerzas de contacto en un } \\
\text { problema. }\end{array}$ \\
\hline 13 & $\begin{array}{l}\text { Estrategia para resolver } \\
\text { problemas de dinámica } \\
\text { y plantear un problema } \\
\text { para resolver en casa so- } \\
\text { bre fuerzas y movimiento }\end{array}$ & 25 & $\begin{array}{l}\text { Que conozcan una posible es- } \\
\text { trategia para resolver proble- } \\
\text { mas y que aprendan a relacionar } \\
\text { la fuerza con la aceleración y el } \\
\text { movimiento. }\end{array}$ \\
\hline 14 & $\begin{array}{l}\text { Resolución de problemas } \\
\text { en clase }\end{array}$ & 40 & $\begin{array}{l}\text { Que conozcan ejemplos de cómo } \\
\text { se pueden aplicar las leyes de } \\
\text { Newton en diferentes tipos de } \\
\text { problemas. }\end{array}$ \\
\hline 15 & $\begin{array}{l}\text { Exposición: ¿Cómo se uti- } \\
\text { lizan para resolver pro- } \\
\text { blemas? (Fuerzas de } \\
\text { rozamiento) }\end{array}$ & 30 & $\begin{array}{l}\text { Que conozcan el concepto de } \\
\text { fuerza de rozamiento. }\end{array}$ \\
\hline 16 & $\begin{array}{l}\text { Resolución de problemas } \\
\text { en clase sobre fuerzas de } \\
\text { rozamiento }\end{array}$ & 40 & $\begin{array}{l}\text { Que aprendan a aplicar la fuerza } \\
\text { de rozamiento en problemas y } \\
\text { que conozcan cómo se resuelven } \\
\text { problemas de mayor dificultad. }\end{array}$ \\
\hline 17 & $\begin{array}{l}\text { Taller: Problema a resol- } \\
\text { ver en parejas }\end{array}$ & 30 & $\begin{array}{l}\text { Que los alumnos trabajen en } \\
\text { equipo y aprendan a argumentar } \\
\text { entre ellos. }\end{array}$ \\
\hline 18 & Cuestionario final & 20 & $\begin{array}{l}\text { Conocer si han aprendido los co- } \\
\text { nocimientos más relevantes del } \\
\text { tema. }\end{array}$ \\
\hline
\end{tabular}

\section{Tabla 2. Desarrollo de las actividades durante el ciclo de mejora.}
$\#$
Desarrollo de la actividad
He pasado un cuestionario de ideas previas para tener una mejor visión
1 de sus conocimientos sobre dinámica y poder ajustar las actividades al resultado. 
He comenzado en clase preguntando porque creían ellos que se movían los cuerpos. Han estado razonando sobre cuáles eran las causas del mo-

2 vimiento, y después de un pequeño debate hemos llegado a la conclusión de que la única causa para cambiar el estado de movimiento de un cuerpo eran las fuerzas.

He realizado un ejemplo en clase para ayudarles a comprender el carácter

3 vectorial que tienen las fuerzas y el significado del principio de superposición de las fuerzas.

Les he planteado la pregunta sobre como pensaban ellos que se producían las fuerzas. Han estado discutiendo sobre cuáles eran los motivos que generaban dichas fuerzas y después he clarificado algunas de sus ideas previas sobre las fuerzas de la naturaleza.

Les he planteado la pregunta de cómo podíamos saber si un cuerpo estaba en movimiento o no, y he definido que era un sistema de referencia inercial.

Hemos visto un ejemplo de aplicación de la 1a ley de Newton y les he plan-

6 teado el problema para resolver en casa de porque si la tierra está en constante movimiento la consideramos como un sistema de referencia inercial.

He preguntado cómo creían ellos que podíamos relacionar las fuerzas con el movimiento y hemos discutido sobre el concepto de masa.

Les he planteado la pregunta de cómo se movían los cuerpos si la 3a ley de

8 Newton parece oponerse a ello. Hemos discutido sobre ello y después he clarificado algunos conceptos.

9 Hemos visto cómo se podían clasificar las fuerzas según su rango de acción y sobre cuáles serían fuerzas de acción a distancia.

Hemos usado el concepto de fuerzas de acción a distancia para calcular el radio de la Tierra mediante el uso de la fuerza gravitatoria.

Hemos seguido con la clasificación de las fuerzas según su rango de ac-

11 ción y hemos hablado sobre los diferentes tipos de fuerzas de contacto que había.

12 Hemos visto un ejemplo del uso de fuerzas de contacto en un problema.

A partir de la resolución de un problema les he mostrado una posible es-

13 trategia para resolver problemas y también hemos visto como relacionar la fuerza con la aceleración y el movimiento.

14 Hemos estado resolviendo diversos problemas y discutiendo algunas de sus características.

Les he preguntado cómo podíamos caracterizar la fuerza de rozamiento

15 y después de estar discutiendo sobre ello, les he clarificado algunos conceptos.

16 Hemos visto algunos problemas dónde se debían considerar las fuerzas de rozamiento y hemos ido resolviendo las dudas que han ido surgiendo. 
17 Les he propuesto un problema para que lo resolvieran por parejas en clase, y razonaran entre ellos.

Para finalizar el ciclo, les he pasado el cuestionario final, que era igual al

18 inicial, con el objetivo de comprobar si habían adquirido los conocimientos deseados al final del tema.

\section{Evaluación del aprendizaje}

Con el objetivo de evaluar el aprendizaje del alumnado durante este ciclo de mejora se ha realizado un análisis de las respuestas del cuestionario inicial y final mostrado con anterioridad (Porlán, 2017). En concreto, el cuestionario inicial lo realizaron 42 alumnos, mientras que el final lo cumplimentaron 37. Las respuestas se han clasificado de la siguiente manera:

D: Respuesta totalmente errónea o sin contestar.

C: Respuesta incorrecta pero el alumno muestra algún conocimiento de la materia.

B: Respuesta parcialmente correcta pero no razonada.

A: Respuesta correcta y razonada

Para la valoración global del cuestionario se ha usado el siguiente valor para cada respuesta: $A=4, B=3, C=2$ y $D=1$.

En particular, el análisis general del cuestionario inicial ha proporcionado los siguientes resultados:

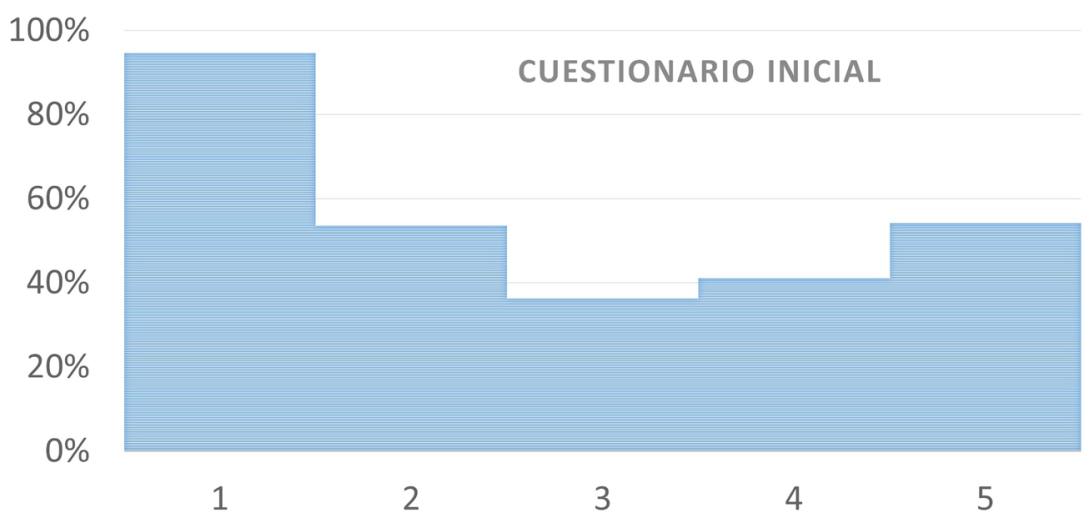

Figura 4. Valoración global de cada pregunta obtenida en el cuestionario inicial.

Jornadas de Formación e Innovación Docente del Profesorado | № 1 (2018) Esta obra se distribuye con la licencia Creative Commons 
En la pregunta 1, que es la que mejor valoración ha obtenido con un $94,6 \%$, se observa que los alumnos parten de un grado de conocimiento elevado y tienen asimilado el concepto de movimiento constante. La pregunta 2, que trataba sobre el concepto de masa, ha obtenido un 53,7\%, mostrando que no todos los alumnos conocen bien dicho concepto. Por otro lado, las preguntas que peor valoración han conseguido son la 3 y la 4, que apenas llegan a un $36 \%$ y a un $41 \%$ respectivamente, y por tanto son las que se deberá prestar especial atención en los ejemplos que se realicen en clase. En concreto, en la pregunta 3 se trata sobre la tercera ley de Newton y por tanto habrá que plantearles algunos ejemplos para que la asimilen. En cuanto a la pregunta 4, se fundamenta en las dos primeras leyes de Newton y en especial en el concepto de que si un cuerpo no experimenta ninguna aceleración entonces dicho cuerpo se encontrará en equilibrio y sobre él la fuerza neta que actuará será nula. A la vista de los resultados, se observa que les cuesta asociar el que haya movimiento y que la fuerza neta sea cero, por tanto, habrá que mostrarles algunos ejemplos donde se les planteen situaciones similares y puedan aprender este concepto. Por último, la pregunta 5 ha obtenido una valoración inicial del 54\%, lo que nos indica que tienen una ida inicial aceptable sobre los conceptos tratados, pero que no los tienen bien asimilados.

Por otro lado, tras el desarrollo del ciclo de mejora se observa, en el análisis general del cuestionario final de la Figura 5, cómo todas las preguntas han obtenido más de un $65 \%$ de puntuación y que, por tanto, las actividades diseñadas han tenido una influencia positiva sobre la evolución de su aprendizaje. En particular, se puede ver cómo la pregunta 1 muestra el porcentaje más alto, como cabía esperar tras el análisis de cuestionario inicial. Además, las preguntas 3 y 4, que eran las que peor valoración habían obtenido en el cuestionario inicial, han mejorado significativamente su puntuación como se describirá más en detalle en la comparación entre el cuestionario inicial y final. 


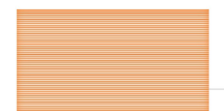

\section{CUESTION}

$60 \%$
$0 \%$
$0 \%$
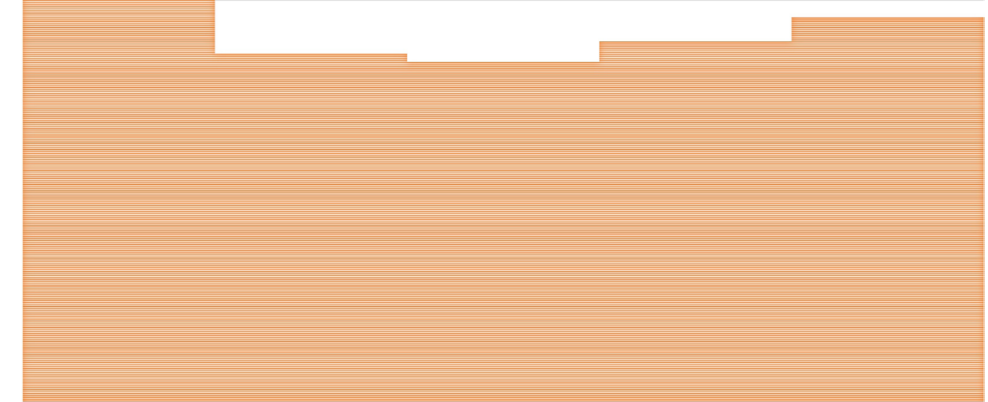

1

2

3

4

5

Figura 5. Valoración global de cada pregunta obtenida en el cuestionario final.

Finalmente, para poder valorar de una forma cuantitativa el progreso realizado por los alumnos durante este ciclo de mejora, se muestra en la Figura 6 una comparativa con la puntuación de cada pregunta en cada cuestionario (azul cuestionario inicial y naranja para el final).

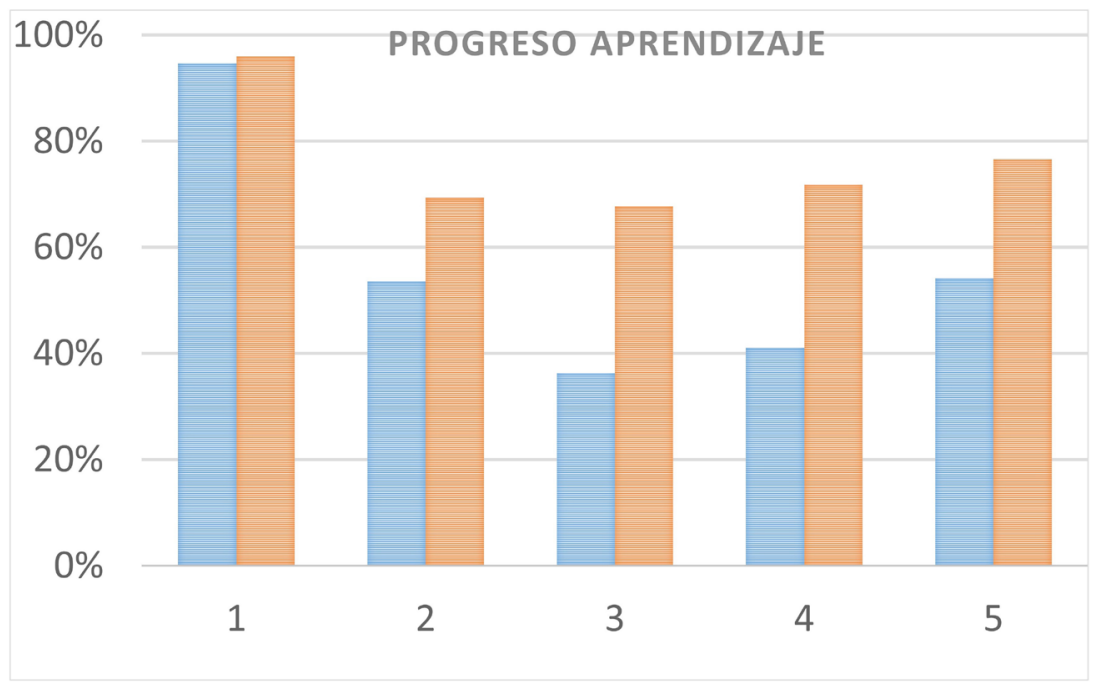

Figura 6. Progreso global del aprendizaje entre el cuestionario inicial (azul) y el final (rojo).

Jornadas de Formación e Innovación Docente del Profesorado | № 1 (2018)

Esta obra se distribuye con la licencia Creative Commons Reconocimiento-NoComercial-SinObraDerivada 
Al comparar la evolución del aprendizaje al inicio y al final del ciclo se observa que en todas las preguntas los alumnos han mejorado significativamente sus resultados previos. En concreto, se puede ver cómo la pregunta 3, que es la que peor resultado había obtenido ha mejorado su puntuación en más de un 30\% y que por tanto los alumnos han mejorado su comprensión sobre la tercera ley de Newton. Por otro lado, la pregunta 4, que también había obtenido una puntuación baja en el cuestionario inicial, ha conseguido una valoración del 72\%, mejorando también en más de un 30\% en relación al cuestionario inicial. El resultado de esta pregunta es significativo, ya que se trataban varios conceptos relacionados con las dos primeras leyes de Newton y requería saber combinarlas, y en función de los resultados se observa cómo han mejorado sus conocimientos respecto a ellos. En la pregunta 1 apenas ha habido mejora ya que se partía de una situación inicial muy favorable. Por último, en las preguntas 2 y 5 también se refleja este progreso del aprendizaje respecto a la situación inicial mejorando alrededor del $20 \%$ respecto al cuestionario inicial.

\section{Evaluación del CMD puesto en práctica}

Mi balance del ciclo es en general positivo, ya que creo que esta forma de enfocar la docencia ha contribuido a su mejora. Además, creo que los estudiantes han desarrollado gran parte de los contenidos que me había marcado al inicio del ciclo. En concreto, en la parte actitudinal, mediante las actividades de contraste se han podido desafiar algunos de sus conocimientos previamente establecidos y de esta manera se ha fomentado un aprendizaje crítico. Por otro lado, el plantearles problemas en clase para realizar en casa, relacionados con el problema del taller a realizar después ha favorecido el aprendizaje continuo.

En cuanto a la parte de contenidos conceptuales y procedimentales desarrollados durante este ciclo destacaría dos aspectos: en primer lugar, creo que la organización de los contenidos mediante mapas y su vinculación a través 
de preguntas clave me ha servido para organizar mejor el tema y para priorizar los contenidos. Además, gracias a ello también he podido diseñar mejor la secuencia de actividades. En segundo lugar, destacaría la utilidad del cuestionario de ideas previas ya que me ha servido para detectar las principales dificultades que tenían los alumnos al inicio del ciclo y con ello he podido orientar las actividades para intentar mejorar dichos aspectos.

También valoro muy positivamente la motivación de los alumnos durante el ciclo, creo que cuando se les planteaban preguntas - y eran ellos los que deducian los conceptos - se les veía más involucrados en clase. También he encontrado muy satisfactoria la realización del taller basado en un problema realizado en parejas. En primer lugar porque han trabajado en grupo y han tenido que razonar algunos de sus conocimientos con sus compañeros y, en segundo lugar, porque les ha valido para replantearse algunos de sus conocimientos previos.

Otro de los aspectos que he encontrado muy útil en el desarrollo del ciclo de mejora ha sido el de la evaluación. creo que el obtener una retroalimentación continuada de su aprendizaje mediante problemas y el uso del cuestionario final me han servido para comprobar de una manera más realista lo que habían aprendido, antes de que pasara demasiado tiempo, y así poder corregir algunas de las deficiencias encontradas.

Por último, ha habido algunos aspectos del ciclo que se podrian mejorar para futuros temas. En concreto, me gustaría aumentar el tiempo dedicado a los talleres y que ésos estuvieran basados en contenidos que todavía no hubiésemos visto en clase. Habría que ver cómo proporcionarles la información previa y ver cómo responden, pero podría ser una buena herramienta para mejorar el aprendizaje. Otro de los aspectos que me gustaría poner en práctica es el de diversificar las clases, plantear actividades distintas, ya sea incluyendo medios audiovisuales o de otro tipo, ya que creo que sería muy beneficioso para evitar la monotonía y estimular a los alumnos. 


\section{Referencias bibliográficas}

Bain, K. (2007). Lo que hacen los mejores profesores universitarios. Valencia: Publicacions de la Universitat de València.

Finkel, D. (2008). Dar clase con la boca cerrada. Valencia: Publicacions de la Universitat de València.

Porlán, R. (Coord.) (2017). Enseñanza Universitaria. Cómo mejorarla. Madrid: Morata/Publicaciones de la Universidad de Sevilla.

Tipler-Mosca, P. (2017). Física para la Ciencia y la Tecnología. Vol. 1. Editorial Reverté. 